\section{Therapy related acute myeloid leukemia with $\mathrm{t}(10: 16)$ : a rare entity}

\author{
Guldeep K. Uppal, ${ }^{1}$ John Leighton, ${ }^{2}$ \\ Deline Da Costa, ${ }^{2}$ Andrew Czulewicz, ${ }^{2}$ \\ Irma E. Palazzo² \\ ${ }^{1}$ Temple University Hospital and 2 Jeanes \\ Hospital, Temple University Health \\ Systems, Philadelphia, USA
}

\section{Abstract}

Treatment related myelodysplastic syndrome (t-MDS) and acute myeloid leukemia (t-AML) are well known complications after chemotherapy for various hematologic and non-hematologic malignancies. Alkylating agents and Topoisomerase inhibitors are most widely studied in this regard. There is growing concern about occurrence of $t$-MDS, $t$ MDS/AML and t-AML in patients of CLL treated with nucleoside analogues especially in combination with alkylating agents. Exact incidence and pathogenesis of nucleoside analogue related MDS/AML is not clear at this time. We hereby report a case of t-AML in a patient treated with Fludarabine, Cyclophosphamide and Rituximab (FCR) for CLL. The cytogenetic studies revealed a unique translocation $t(10: 16)$, that has been reported in very few cases of therapy related AML and pediatric AML.

\section{Introduction}

Occurrence of second malignancies is a welldocumented phenomenon in chronic lymphocytic leukemia (CLL). Disease related immune deficiency and decreased immunosurveillance is purposed as one of the mechanisms related to these second malignancies. ${ }^{1}$ Recent advances in CLL treatment have led to better remission rates and prolonged survival in CLL patients. At the same time treatment related late onset toxicities such as therapy related t-MDS, MDS /AML and AML have been reported in some cases. We hereby report a case of t-AML with t (10:16) arising in patient treated with FCR regimen for CLL. To the best of our knowledge this is the first case of t-AML with $t$ (10:16) in a patient of CLL treated with Fludarabine and Cyclophosphamide.

\section{Case Report}

This patient is an 83-year-old male who was diagnosed with CLL in 2007. He received six cycles of Fludarabine, Cyclophosphamide and Rituximab from September 2007 to March 2008. Due to his co-morbities, attenuated doses were used and a $10 \%$ dose reduction was done for Fludarabine and Cyclophosphamide. Off therapy, he had stable cytopenias in all three cell lines. In June 2011, he presented with increasing White Blood Cell (WBC) count and thrombocytopenia. A clinical differential diagnosis of CLL relapse versus a prolymphocytic transformation versus myelodysplastic syndrome was made. At the time of presentation his $\mathrm{CBC}$ findings were as follows: WBC count 30.7/MM3, hemoglobin $9.4 \mathrm{gm} / \mathrm{dL}$ and platelet count 19/KMM3. The peripheral blood smear showed numerous small lymphoid cells along with some large immature cells. These immature cells showed nuclei with fine nuclear chromatin, 1-3 prominent nucleoli and mild to moderate amount of blue grey cytoplasm. Some of the cells showed nuclear indentation, nuclear membrane folding and fine cytoplasmic vacuolation. On flow cytometry, two distinct populations were observed; one in the lymphocytic gate and the second in the monocytic gate (Figure 1). The population in the lymphocytic gate showed co-expression of CD19, CD20, CD5, CD23 and kappa light chains. The cell population in the monocytic gate was positive for expression of CD2, CD4, CD33 and CD64. These cells showed lack of expression of CD14, CD16, CD34, CD117 and HLA-DR. The bone marrow aspirate smears were predominantly composed of blasts (50\% of the all nucleated cells) and mature lymphoid cells (30\% of the all nucleated cells) (Figure 2). The bone marrow biopsy findings included a hypercellular marrow with marked suppression of trilineage hematopoiesis and aggregates of mature looking lymphocytes amidst sheets of immature blasts (Figure 3). This case was diagnosed as AML with monocytic features arising in background of CLL. He was started on Azacytidine then. The cytogenetic findings were as follows: 46XY, $\operatorname{der}(4) \mathrm{t}(4: 16)(\mathrm{p} 14: \mathrm{q} 22), \quad \operatorname{inv}(6)$ (p23:q15), $\mathrm{t}(10: 16)$ (q22:p13.3), del(20q) (q11.2q13.1). $t(10: 16)$ and $\operatorname{del}(20 q)$ were observed in all twenty metaphases analyzed.

\section{Discussion}

The clinical course of CLL can be complicated by transformation to a prolymphocytic leukemia, diffuse large B-cell lymphoma (Richter's transformation) and rarely even acute lymphoblastic leukemia. ${ }^{1}$ With the recent advances in the treatment of NonHodgkin Lymphomas, the remission and overall survival rates have markedly improved in patients with CLL. With prolonged survival, the
Correspondence: Irma E. Palazzo, Department of Pathology and Laboratory Medicine, Jeanes Hospital, 7604 Central Avenue, 19111, Philadelphia, USA.

Tel. +1.215.728.2347.

E-mail: irma.palazzo@tuhs.temple.edu

Key words: acute myeloid leukemia, myelodysplastic syndrome.

Acknowledgements: we acknowledge and appreciate the cooperation of our patient and his family.

Contibutions: GU, case report conceiving, literature searching, manuscript writng; JL, patient evaluation, text reviewing; IP, histopathological data analysis, text reviewing; DD, data analysis, text reviewing; $\mathrm{AC}$, final text reviewing.

Conflict of interest: the authors report no conflicts of interest.

Received for publication: 26 September 2011. Accepted for publication: 12 October 2011.

This work is licensed under a Creative Commons Attribution NonCommercial 3.0 License (CC BYNC 3.0).

(C) Copyright G.K. Uppal et al., 2011

Licensee PAGEPress, Italy

Hematology Reports 2010; 3:e23

doi:10.4081/hr.2011.e23

late effects of therapy are becoming more evident and hence more amenable to study.

Two subtypes of therapy related myeloid neoplasms (t-MN) are described in WHO classification of hematopoietic neoplasms. The $t-$ MN occurring after administration of alkylating agents occurs after long latent period of 510 years; frequently present with cytopenias, myelodysplasia and abnormalities of chromosome 5 and 7. On the other hand t-MN related to the Topoisomerase Inhibitors frequently present with overt leukemia without preceding MDS and is more commonly associated with balanced translocations. ${ }^{2}$ t-MN related to purine analogues such as Fludarabine and Cladribine have been infrequently reported. Accurate incidence and clinicopathologic features of this group of patients are not well described in the literature.

The relative contributions of chemotherapeutic interventions in the onset of $t$ MDS/AML associated with nucleoside analogues are not well defined. Some authors have reported increased risk of MDS/AML when nucleoside analogues are used in combination with alkylating agents. ${ }^{3}$ Fludarabine has been reported to augment the cytotoxic damage caused by alkylating agents. ${ }^{4}$

The CALGB9011 study reported an incidence of $3.5 \%$ in patients who were treated with both Fludarabine and Chlorambucil, $0.5 \%$ with Fludarabine alone and $0 \%$ in Chlorambucil 
alone. ${ }^{5}$ Recently Kluk et al. reported a series of five patients of t-MDS/AML (MDS=3, AML=2) who were treated with Fludarabine $(n=4)$ and Fludarabine with rituximab $(\mathrm{n}=-1)$ for CLL $(\mathrm{n}=4)$ and marginal zone lymphoma $(\mathrm{n}=1)$. They compared these patients to a control group of 15 patients who were only treated with alkylating agents and reported a short latency period, less likely tendency to show complex karyotypes and longer median sur-

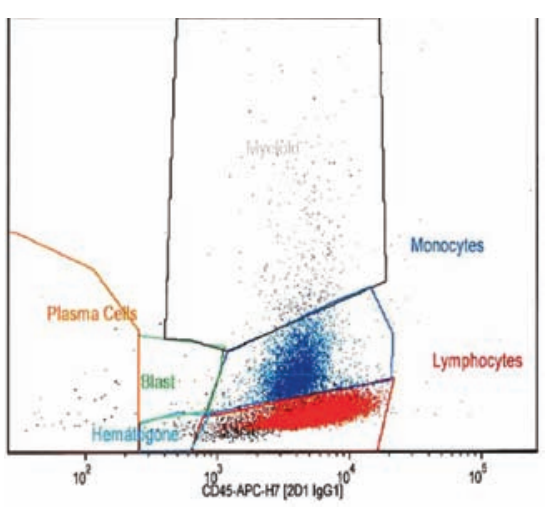

Figure 1. Open gated scattergram showing two distinct populations; one in the monocytic gate (blue) and the second in the lymphocytic gate (red).

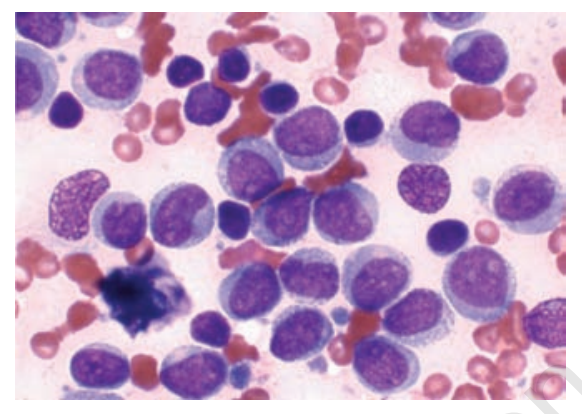

Figure 2. Bone marrow aspirate smears showing large blasts with monocytic features and few small lymphomatous cells in the background, Wright-Giemsa, 100X.

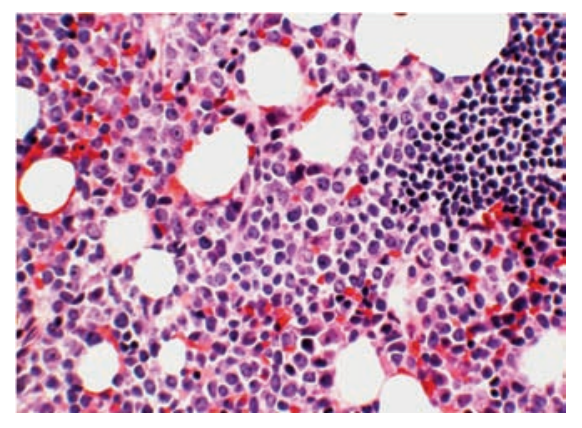

Figure 3. Hypercellular bone marrow biopsy showing involvement by sheets of blasts and neoplastic lymphoid cells, Hematoxylin \& Eosin, 400X. vival in patients treated with Fludarabine alone. ${ }^{6}$ Čolović et al. reported retrospective analysis of 210 CLL patients who were treated with Fludarabine and Cyclophosphamide. They reported t-MDS/AML in 4/210 (1.9\%) patients which is slightly less than in other studies. ${ }^{7}$ Tam et al reported results of FCR (Fludarabine, Cyclophosphamide and Rituximab) treatment for CLL. Out of 300 patients, 8 patients developed MDS/AML. ${ }^{8}$

The risk factors for developing $\mathrm{t}$-AML or MDS or MDS/AML are not well defined. There is emerging role of genetic predisposing factors. Larson has previously reported increased frequency of NQ01 gene polymorphisms in patients with t-MN. ${ }^{9}$ Other reported risk factors for occurrence of $\mathrm{t}$-MDS/AML in CLL patients include: paratrabecular pattern of bone marrow infiltration by lymphoma, treatment with Rituximab, prolonged bi/pan cytopenias and hypocellular marrow after Fludarabine treatment. ${ }^{10}$

Various cytogenetic complex karyotypes have been reported in t-MDS/AML in CLL. The most frequently reported are del (5) (q13q33), del (7), +3mar (cp20), +mar (13) and multiple other aberrations of chromosome 8, 11, 15 and 17. ${ }^{11}$ Cytogenetic findings in our patient revealed del $(20 q)$ and an unusual findings of $\mathrm{t}(10: 16)$.

While del (20q) is a common findings in tMDS/AML, t (10:16) is rare rearrangement that has been reported in very few cases of AMLM4/M5. ${ }^{12}$ In $t$ (10:16), the monocytic leukemia Zinc finger protein related factor (MORF) at $10 \mathrm{q} 22$ has been shown to fuse with CREB binding protein (CBP) gene at $16 \mathrm{p} 13$. MORF resembles monocytic leukemia zinc finger (MOZ) gene. ${ }^{12,13}$ Cases of MOZ-CBP fusion $\mathrm{t}$ (8:16) have shown to be associated with monocytic differentiation. ${ }^{14}$ However role of MORFCBP fusion gene in leukemogenesis is not well studied in the literature. To the best of our knowledge only 5 cases have been reported so far. Pebusque et al reported a case of AML-M4 in patient with two reciprocal translocations involving $t$ (11:17) and $t$ (10:16). ${ }^{15}$ Later on Panagopoulos et al reported a case of AML-5a in a 4-year-old girl with a complex karyotype including $t$ (10:16)(q22;p13). ${ }^{16}$ Another case of AML with t (10:16) was reported in an 84-yearold male with AML-M4. ${ }^{17}$ Interestingly Kojima et al reported a case of 52-year-old female who was diagnosed with AML with $\mathrm{t}$ (10:16) after prolonged preleukemic phase and the blasts did not show evidence of monocytic differentiation. ${ }^{18}$

\section{Conclusions}

Risk of therapy related MDS or AML is an emerging problem in era of successful treatment of many hematologic as well as nonhematologic malignancies. Our case is interesting and educational at several levels. In patients of CLL treated with nucleoside analogues and or alkylating agents, who present with various cytopenias or suspected transformation, a differential diagnosis of t-MN should be kept in mind. This case also demonstrated presence of a rare translocation, $t$ (10:16). This finding might be helpful in understanding the pathogenesis of t-MN.

\section{References}

1. Ricci F, Tedeschi A, Montillo M, Morra E. Therapy related myeloid neoplasms in chronic lymphocytic leukemia and Waldenström's macroglobulinemia. Mediterr J Hematol Infect Dis 2011;3: e2011031.

2. Vardiman, Thiele J, Arber DA et al. The 2008 revision of the World Health Organization (WHO) classification of myeloid neoplasms and acute leukemia: rationale and important changes. Blood 2009;114:937-51.

3. Rai KR, Peterson BL, Appelbaum FR et al. Fludarabine compared with chlorambucil as primary therapy for chronic lymphocytic leukemia. N Engl J Med 2000;343:17507.

4. Yamauchi T, Nowak BJ, Keating MJ, Plunkett W. DNA repair initiated in chronic lymphocytic leukemia lymphocytes by 4hydroperoxycyclophosphamide is inhibited by fludarabine and clofarabine. Clin Cancer Res 2001;7:3580-9.

5. Morrison VA, Rai KR, Peterson BL et al. Therapy-related myeloid leukemias are observed in patients with chronic lymphocytic leukemia after treatment with fludarabine and chlorambucil: results of an intergroup study, cancer and leukemia group B 9011. J Clin Oncol 2002;20:387884.

6. Kluk M, Toomey C, Hochberg E, Hasserjian R. Myelodysplastic syndromes following therapy with purine analogues - Therapy related myeloid neoplasms? (Poster Presentation) USCAP annual meeting 2009. Available from: http://www.abstracts2view.com/ uscap09/view.php?nu=USCAP09L_1234

7. Čolović M, Suvajdžić N, Janković G et al.Therapy-related myelodysplastic syndrome and acute myeloid leukemia in patients with chronic lymphocytic leukemia treated with fludarabine and cyclophosphamide. Biomed Pharmacoter 2011;65:319-21.

8. Tam CS, O'Brien S, Wierda W et al. Long- 
term results of the fludarabine, cyclophosphamide, and rituximab regimen as initial therapy of chronic lymphocytic leukemia. Blood 2008;112:975-80.

9. Larson RA, Wang Y, Banerjee $\mathrm{M}$ et al. Prevalence of the inactivating $609 \mathrm{C}-->\mathrm{T}$ polymorphism in the NAD(P) H:quinone oxidoreductase (NQ01) gene in patients with primary and therapy-related myeloid leukemia. Blood 1999;94:803-7.

10. Carney DA, Seymour JF. Therapy-related myelodysplasia and fludarabine combination therapy - do the benefits justify the risk? Leuk Lymphoma 2010;51:1957-9.

11. Tam CS, Seymour JF, Prince HM et al. Treatment-related myelodysplasia following fludarabine combination chemotherapy. Haematologica 2006;91:1546-50.
12. Vizmanos JL. $\mathrm{t}(10 ; 16)(\mathrm{q} 22 ; \mathrm{p} 13)$. Atlas Genet Cytogenet Oncol Haematol. May 2006. Available from: http://atlasgeneticsoncology.org/Anomalies/t1016q22p13ID13 32.html

13. Katsumoto T, Yoshida N, Kitabayashi I. Roles of the histone acetyltransferase monocyticleukemia zinc finger protein in normal and malignant hematopoiesis. Cancer Sci 2008;99:1523-7.

14. Quesnel B, Kantarjian H, Bjergaard JP et al. Therapy-related acute myeloid leukemia with $\mathrm{t}(8 ; 21)$, inv(16), and $t(8 ; 16)$ : a report on 25 cases and review of the literature. J Clin Oncol 1993;11:2370-9.

15. Pébusque MJ, Lafage $M$, Lopez $M$, Mannoni P. Preferential response of acute myeloid leukemias with translocation involving chromosome 17 to human recombinant granulocyte colony-stimulating factor. Blood 1988;72:257-65.

16. Panagopoulos I, Fioretos T, Isaksson M et al. Fusion of the MORF and CBP genes in acute myeloid leukemia with the $\mathrm{t}(10 ; 16)(\mathrm{q} 22 ; \mathrm{p} 13)$. Hum Mol Genet 2001;10:395-404.

17. Vizmanos JL, Larrayoz MJ, Lahortiga I et al. $\mathrm{t}(10 ; 16)(\mathrm{q} 22 ; \mathrm{p} 13)$ and MORF-CREBBP fusion is a recurrent event in acute myeloid leukemia. Genes Chromosomes Cancer 2003;36:402-5.

18. Kojima K, Kaneda K, Yoshida C et al. A novel fusion variant of the MORF and CBP genes detected in therapy-related myelodysplastic syndrome with $\mathrm{t}(10 ; 16)$ (q22;p13). Br J Haematol 2003,120:271-3. 